\title{
Dissociable effects of cocaine and yohimbine on impulsive action and relapse to cocaine seeking
}

\author{
Nienke Broos $^{1}$ - Yvar van Mourik ${ }^{1}$ Dustin Schetters ${ }^{1} \cdot$ Taco J. De Vries $^{1}$ • \\ Tommy Pattij ${ }^{1}$
}

Received: 10 April 2017 / Accepted: 6 August 2017 / Published online: 30 August 2017

(C) The Author(s) 2017. This article is an open access publication

\begin{abstract}
Rationale A strong association has been demonstrated between various forms of impulsivity and addiction-like behavior in both humans and rats.

Objectives In this study, we investigated how impulsive action, as measured in the 5-choice serial reaction time task (5CSRTT), is affected during various stages of cocaine taking and seeking and by relapse-provoking stimuli in animals that were trained both in an intravenous cocaine self-administration paradigm and in the 5-CSRTT.

Methods Rats were concurrently trained in the 5-CSRTT and cocaine self-administration protocol, and subsequently, the effects of cocaine $(7.5 \mathrm{mg} / \mathrm{kg})$ and the pharmacological stressor yohimbine $(1.25 \mathrm{mg} / \mathrm{kg})$ were tested in both paradigms. Results Cocaine self-administration $(5 \mathrm{~h} /$ day) transiently altered impulsive action and increased errors of omission in the 5-CSRTT. Pharmacological challenges with cocaine and yohimbine induced increments in impulsive action and reinstated cocaine-seeking responses within the same animals. Further analyses revealed that the effects of cocaine and yohimbine on impulsive action did not correlate with their effects on reinstatement of cocaine seeking.

Conclusions These data suggest that although impulsive action and relapse can be pharmacologically modulated in the same direction within individuals, these effects appear not to be directly coupled.
\end{abstract}

Taco J. De Vries and Tommy Pattij shared senior authorship.

Tommy Pattij

t.pattij@vumc.nl

1 Department of Anatomy and Neurosciences, Amsterdam Neuroscience, VU University Medical Center, De Boelelaan 1108 , $1081 \mathrm{HZ}$ Amsterdam, The Netherlands
Keywords Impulsivity · Cocaine self-administration . Relapse · Yohimbine

\section{Introduction}

There is broad consensus that drug dependence and impulsivity are closely related. For instance, clinically there is high comorbidity between attention-deficit/hyperactivity disorder (ADHD) and substance-use disorders (Van Emmerik-van Oortmerssen et al. 2012). Maladaptive impulsivity is a key symptom in ADHD (Moeller et al. 2001) and, moreover, elevated impulsivity is also frequently observed in substance dependence, including cocaine dependence (for reviews, see De Wit 2009; VerdejoGarcia et al. 2008; Pattij and De Vries 2013). Additionally, preclinical animal studies have unequivocally demonstrated that impulsive rats are more vulnerable to several measures of addiction-like behavior (Belin et al. 2008; Diergaarde et al. 2008; Dalley et al. 2007; Broos et al. 2015; Jupp et al. 2013; Perry and Carroll 2008; Winstanley et al. 2010).

Impulsivity is widely viewed as action without adequate forethought and consists of dissociable behavioral modalities (Broos et al. 2012b; Evenden 1999; Winstanley et al. 2004). These dissociable modalities of impulsivity show distinct relationships with various phases of drug addiction (for reviews, see Winstanley et al. 2010, Pattij and De Vries 2013). One of these modalities, impulsive action, arises from deficient inhibitory-response control and relates to an elevated risk to the escalation of compulsive cocaine intake (Belin et al. 2008; Dalley et al. 2007). In addition, high levels of impulsive action are associated with a stronger cue-induced reinstatement response after punishment-induced abstinence (Economidou et al. 2009). Together these studies highlight that impulsivity co-exists with high risk to addictive-like behavior, yet do not necessarily imply that impulsivity mediates this risk. 
One important mediator of relapse in drug dependence is stress, and over the last two decades, translational models have tremendously contributed to our understanding of the neural correlates of stress-induced relapse (for a recent review, see Mantsch et al. 2016). A variety of stressors are able to reinstate drug seeking, among which is the pharmacological stressor yohimbine (Charney et al. 1984; Shepard et al. 2004). Besides cues and stress, relapse to cocaine seeking can be reliably provoked by priming injections of cocaine (for a review, see Bossert et al. 2005). Interestingly, cocaine and yohimbine have also been demonstrated to induce an increase in impulsive action (Schippers et al. 2016; Sun et al. 2010; Torregrossa et al. 2012; van Gaalen et al. 2006; Winstanley et al. 2007). Despite the fact that yohimbine and cocaine have similar effects on relapse propensity and impulsive action, it remains to be studied whether overlapping mechanisms are responsible for this, or whether independent mechanisms underlie these effects.

The present study aims to explore the interrelationship between impulsive action and cocaine relapse by continuous monitoring of impulsivity levels during various stages of long-access cocaine taking and seeking. Additionally, the acute effects of cocaine and yohimbine on impulsive action and reinstatement of cocaine seeking were directly compared using a within-subject design.

\section{Materials and methods}

\section{Animals}

Male Wistar rats (Harlan, Horst, The Netherlands), initially weighing 250-270 g, were housed in standard Macrolon cages on a reversed 12-h day/night cycle (lights on, 7 p.m.) in a temperature $\left(21 \pm 2{ }^{\circ} \mathrm{C}\right)$ and humidity $(50 \pm 10 \%)$ controlled room. Rats were housed in pairs until surgery, and individually afterwards. Behavioral testing was conducted during the dark phase of the day/night cycle. During the entire experiment, rats were food restricted and maintained at about 85-90\% of their free-feeding weight by providing them with 14-18 $\mathrm{g}$ of chow at the end of each day. Water was available ad libitum. Experiments were approved by the Animal Care committee of the VU University and VU University medical center, Amsterdam, The Netherlands.

\section{Five-choice serial reaction time task (5-CSRTT)}

Detailed descriptions of apparatus and training procedures have been provided previously (Van Gaalen et al. 2006). In short, the final procedure of the 5-CSRTT was as follows: rats were placed in an operant chamber containing a food receptacle and an array of five rectangular apertures in the opposing wall. After starting the trial by a nose poke in the receptacle, they were required to wait for $5 \mathrm{~s}$ (inter-trial interval, ITI) before one of the stimulus lights within the apertures was illuminated for $1 \mathrm{~s}$. A nose-poke response into this illuminated hole was rewarded with one food pellet. Every session consisted of 100 trials or lasted $30 \mathrm{~min}$, whichever occurred first. The following behavioral measures were recorded to assess task performance: (1) accurate choice, i.e., percentage correct responses calculated as [number correct trials/(correct + incorrect trials) $] \times 100$ as a measure of visuospatial attention; (2) premature responses as a measure of impulsive action, i.e., number of responses into any of the holes during the ITI period and before stimulus onset; (3) number of omissions, i.e., number of omitted trials during a session as a measure of motivation and/or motor performance; (4) perseverative responses after correct choice, as a measure of inhibition related to compulsive-like response patterns; (5) correct response latency, i.e., the mean time between stimulus onset and a correct response as a measure of speed of responding and/or motor performance; and (6) feeder latency, i.e., the latency to collect a pellet following a correct choice as a measure of motivation.

\section{Cocaine self-administration}

Detailed description of apparatus and training procedures has been provided previously (Broos et al. 2012a). Briefly, operant two-lever chambers were equipped with a red house light, a white noise generator, and a liquid swivel connecting rats to an infusion pump (total volume of $42.52 \mu$ delivered over $2 \mathrm{~s}$ ). Rats were randomly assigned to one of two different contexts. These contexts differed in (1) white noise $(70 \mathrm{~dB})$, either continuous or interval; (2) odor, either lemon-scented or almond-scented; and (3) the chamber floor, a flat PVC surface with either holes or straight grooves. The employed cocainetaking and cocaine-seeking paradigm started with acquisition of cocaine (cocaine-HCl, OPG, Utrecht, The Netherlands) self-administration under a fixed-ratio 1 (FR1) schedule of reinforcement and occurred in daily 5-h sessions in the designated context. To reduce the anxiogenic effects of cocaine, rats received $250 \mu \mathrm{g} / \mathrm{kg} /$ infusion of cocaine during the first five sessions, followed by $500 \mu \mathrm{g} / \mathrm{kg} /$ infusion for the remaining sessions. To prevent overdosing in inexperienced rats, the maximum number of rewards was set to 40 rewards for the first three sessions and to 100 rewards for the next two sessions. After this, animals could earn an unlimited number of infusions. There was a non-signaled time-out of $15 \mathrm{~s}$ after every infusion. Inactive lever presses were registered, but without consequences. Subsequently, following acquisition, responding under a progressive ratio schedule of reinforcement was assessed in four subsequent identical 4-h sessions to assess the motivation to self-administer cocaine. During progressive ratio responding for cocaine, the response ratio requirement and number of active responses resulting in a 
cocaine infusion was progressively increased between infusions following the equation response ratio $=5 \times e^{(0.2 \times \text { infusion }}$ number) -5 , rounded to the nearest integer.

Extinction of cocaine self-administration was measured in two different types of extinction procedures. First, to test the effects of exposure to the drug-associated context on reinstatement of cocaine seeking, operant extinction training took place to extinguish responses on the active lever. This extinction procedure consisted of daily 1-h sessions in a context different from the cocaine SA context, in which no odor was introduced, white noise was absent, and there was a grid floor. During operant extinction training, all responses were registered, but without programmed consequences. Following operant extinction, a context-induced reinstatement test was conducted by exposing animals to the cocaine-associated context to determine whether this would elicit drug-seeking responses. Subsequently, a second extinction procedure was followed by subjecting the animals to context extinction to allow assessment of the effects of cocaine and yohimbine on reinstatement of drug-seeking responses. During context-extinction sessions which lasted $1 \mathrm{~h}$, the cocaine-associated context was reintroduced into the operant chamber (white noise, odor, and PVC floor). All responses were registered, but without consequences. Following context extinction, a cocaineinduced reinstatement test was performed under contextextinction conditions that lasted $30 \mathrm{~min}$. During this reinstatement test, $20 \mathrm{~min}$ prior to testing, all rats received intraperitoneal injections of saline ( $1 \mathrm{ml} / \mathrm{kg}$ bodyweight) on the first test day and cocaine $(7.5 \mathrm{mg} / \mathrm{kg})$ on the following test day. Subsequently, five additional context-extinction sessions were given followed by a stress-induced reinstatement test using yohimbine as a pharmacological stressor. For this, rats received an acute challenge with the $\alpha 2$-adrenoceptor antagonist yohimbine $(1.25 \mathrm{mg} / \mathrm{kg}$, intraperitoneal, $1 \mathrm{ml} / \mathrm{kg}$ bodyweight; Sigma, St. Louis, MO, USA) 45 min prior to the reinstatement test. Rats received sterile water $(1 \mathrm{ml} / \mathrm{kg}$ bodyweight) on the first test day and yohimbine on the following test day.

\section{Experimental design}

The current experiment was designed to explore whether the co-occurrence between impulsive action and relapse to cocaine seeking is due to interrelated phenomena. To that end, we compared direct manipulations of impulsive action with direct manipulations of reinstatement to drug seeking in the same animals using a within-subject design (Fig. 1). A cohort of 32 rats was trained in the 5-CSRTT and upon stable baseline performance equipped with jugular catheters. Upon reestablishment of stable 5-CSRTT performance, all rats were trained in the afternoon to self-administer cocaine under long-access $(5 \mathrm{~h})$ conditions. The following behavioral stages of cocaine taking and cocaine seeking were studied: (1)
5-CSRTT training: $56 \mathrm{~d}$

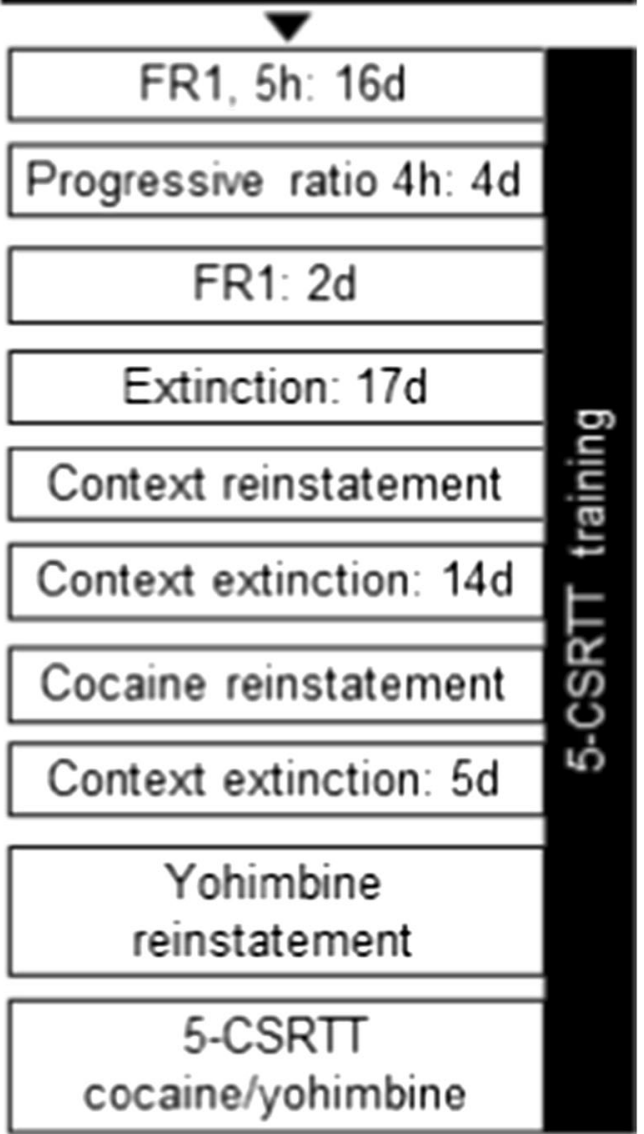

Fig. 1 Schematic diagram depicting the design and order of the experiments

cocaine self-administration on FR1 schedule (16 sessions), (2) progressive ratio responding (four sessions), (3) operant extinction (17 sessions), (4) context-induced reinstatement, (5) context extinction (14 sessions), and (6) cocaine- and yohimbine-induced reinstatement. Subsequently, 1 week following these tests, the effects of acute cocaine and yohimbine challenges on 5-CSRTT performance (including impulsive action) were tested. First, acute effects of cocaine/saline were tested, and a week later, acute effects of yohimbine/water were tested. In the 5-CSRTT, the drugs and their respective vehicles were tested in counterbalanced fashion within individuals across both testing days which were Tuesdays and Thursdays with baseline training sessions on other weekdays. Dosing and timing of injections were identical to the reinstatement tests. During the entire cocaine SA paradigm, rats were trained in the 5-CSRTT every morning on weekdays, and the presented data of concurrent 5-CSRTT sessions show the performance averaged per week similar to previous work (Winstanley et al. 2009). Due to the loss of catheter patency in the course of the experiments, $n=9$ animals were excluded, leaving $n=23$ animals in all analyses unless stated otherwise. 


\section{Statistical analyses}

Data were analyzed using repeated measures analysis of variance (ANOVA) and, in the case of the pharmacological challenges, a two-way repeated measures ANOVA with dose (vehicle or dose) and drug (cocaine or yohimbine) as withinsubjects variable. For the ANOVAs, the homogeneity of variance was determined using Mauchly's tests for equal variances, and in the case of violation of homogeneity, Huynh-Feldt epsilon $(\varepsilon)$ adjusted the degrees of freedom; the resulting more conservative probability values were depicted and used for subsequent analyses. For SA data, sessions and lever (active vs inactive) served as within-subjects variables and the number of active and inactive responses were dependent variables. For the 5-CSRTT data, the dependent variables were the number of premature responses, accuracy, number of omissions, perseverative responses, and correct response and feeder latencies. Pearson's correlation analyses were used to test whether reactivity to cocaine and yohimbine compared to their respective vehicle in the 5-CSRTT and reinstatement paradigms was related. Data were analyzed using the Statistical Package for the Social Sciences version 20.0 (SPSS, Chicago, IL, USA), and the significance level was set at $p<0.05$.

\section{Results}

\section{Cocaine self-administration paradigm}

All rats readily acquired cocaine self-administration over the course of 16 sessions by increments in active lever responding to the level of approximately 80 cocaine infusions per session, whereas responding on the inactive lever did not significantly change [Fig. 2a; active responses: $F(15,330)=29.95$, $p<0.001, \varepsilon=0.36$; inactive responses: $F(15,330)=1.96$, $\varepsilon=0.50, \mathrm{NS}]$. Following this, responding for cocaine under a progressive ratio schedule of reinforcement increased the levels of active responding but not the inactive responding over the different ratios during four subsequent days [Fig. 2b; active responses: $F(3,66)=3.92, \varepsilon=0.54, p=0.037$; inactive responses: $F(3,66)=1.17, \varepsilon=0.68, \mathrm{NS}]$. After progressive ratio responding, animals were subjected to two additional FR1 sessions (data not shown), and thereafter during 17 subsequent sessions, operant responding for cocaine was extinguished in a neutral context and all rats rapidly decreased responding on both the active lever and inactive lever [Fig. 2c; active responses: $F(16,352)=13.90$, $\varepsilon=0.20, p<0.001$; inactive responses: $F(16,352)=4.03$, $\varepsilon=0.50, p<0.001]$ in the absence of cocaine. Exposure of the rats to the cocaine-associated context reinstated responding on the active lever that subsequently decreased over 14 sessions of context extinction training to the cocaine-associated context [Fig. 2d; active responses: $F(13,286)=11.49, \varepsilon=0.42, p<0.001$; inactive responses: $F(16,352)=4.03, \varepsilon=0.50, p<0.001$ ].

\section{5-CSRTT performance during the cocaine self-administration paradigm}

Concurrent training in the 5-CSRTT during cocaine SA revealed that during acquisition and progressive ratio responding for cocaine, rats became less impulsive in the last two weeks of selfadministration compared to the first week [Fig. 3a; $F(3,66)=3.53, \varepsilon=0.80, p=0.029]$. In contrast, over the course of operant extinction learning, premature responses significantly increased during week 4 compared to the other three weeks of
Fig. 2 Measures of cocaine taking and seeking behavior in all included $n=23$ animals. a Number of cocaine infusions/ responses during cocaine selfadministration under an FR1 schedule of reinforcement. b Number of responses under progressive ratio responding for cocaine. $\mathbf{c}$ Number of responses during operant extinction of cocaine seeking. $\mathbf{d}$ Number of responses during context extinction of cocaine seeking in the cocaine-associated context
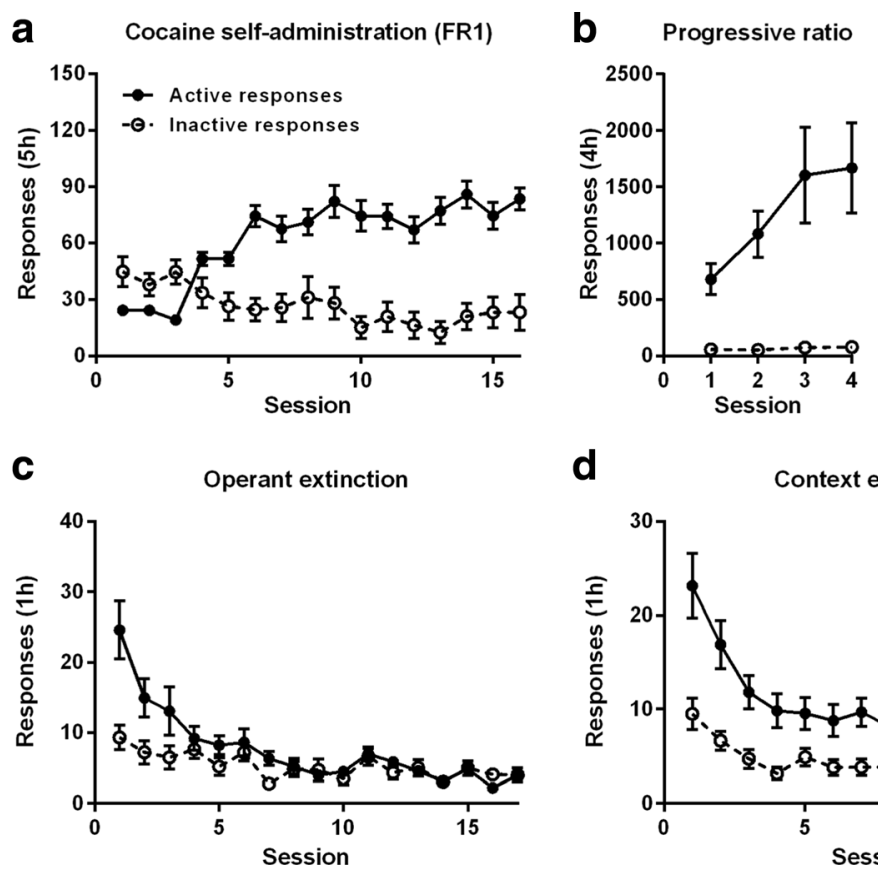

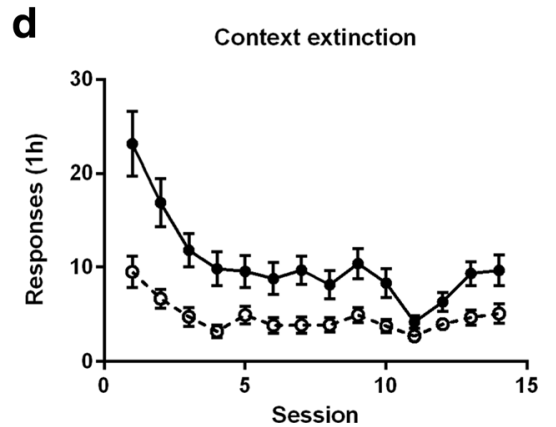


the operant extinction procedure $[F(3,66)=3.89, \varepsilon=0.72$, $p=0.025]$, whereas during context extinction, premature responding did not change $[F(2,44)=1.70, \mathrm{NS}]$. Likewise, perseverative responding after correct choice also significantly decreased during week 3 of cocaine SA compared to the first week [Fig. 3b; $F(3,66)=3.1, \varepsilon=0.73, p=0.048]$ and increased during weeks 3 and 4 of operant extinction learning compared to week $1[F(3,66)=5.84, \varepsilon=0.76, p=0.004]$ yet did not change over the course of context extinction $[F(2,44)=3.27, \varepsilon=0.77$, NS] Levels of accurate choice were neither affected during acquisition and PR responding for cocaine SA [Fig. $3 \mathrm{c} ;[F(3,66)=1.53$, $\varepsilon=0.59, \mathrm{NS}]$ nor during operant extinction learning $[F(3,60)=0.94, \varepsilon=0.42, \mathrm{NS}]$ and context extinction learning $[F(2,42)=1.10, \mathrm{NS}]$. Importantly, $n=2$ animals consistently omitted all trials within a 5-CSRTT session over the entire operant extinction learning period. Of these $n=2$ animals, one animal maintained omitting all trials over the contextextinction period. The number of omitted trials, significantly increased during weeks 2 and 3 compared to week 1 of cocaine SA and progressive ratio responding [Fig. $3 \mathrm{~d} ; F(3,66)=5.08$, $\varepsilon=0.82, p=0.006]$, whereas during operant extinction learning, the number of omissions significantly decreased over weeks $[F(3,66)=12.62, \varepsilon=0.46, p<0.001]$, which remained stable over context extinction learning $[F(2,44)=0.93$, NS $]$. Response latencies were shortened in week 4 compared to weeks 2 and 3 during cocaine SA and progressive ratio responding [Fig. 3e; $F(3,66)=4.92, \varepsilon=0.80, p=0.008]$, and also, during weeks 3 and 4 of operant extinction learning, response latencies were shortened compared to the first week of extinction $[F(3,60)=3.18, \varepsilon=0.71, p=0.049]$ and were lengthened during the final week of context extinction compared to the first week $[F(2,42)=3.78, p=0.031]$. Finally, feeder latencies were altered neither during cocaine $\mathrm{SA}$ and progressive ratio responding [Fig. 3f; $F(3,60)=2.33, \varepsilon=0.38, \mathrm{NS}$ ] nor during operant extinction learning $[F(3,60)=2.96, \varepsilon=0.37$, NS $]$ and context extinction learning $[F(2,44)=0.37, \varepsilon=0.69$, NS $]$.

\section{Cocaine and yohimbine effects on reinstatement to cocaine seeking and 5-CSRTT performance}

Following the context-induced reinstatement test and extinction of the cocaine-associated context, acute effects of $7.5 \mathrm{mg} / \mathrm{kg}$ cocaine and $1.25 \mathrm{mg} / \mathrm{kg}$ yohimbine were tested on reinstatement of cocaine seeking. During these tests, both cocaine and yohimbine strongly increased the number of active lever responses [Fig. 4a; dose: $F(1,22)=20.85, p<0.001$; drug: $F(1,22)=3.84$, NS; drug $\times$ dose: $F(1,22)=2.37$, NS]. In addition, the number of inactive responses during
Fig. 3 Concurrent training in the 5-CSRTT during cocaine taking and seeking behavior. Impulsive action (a) and the number of perseverative responses (b) decreased during cocaine selfadministration and increased during operant extinction training. Visuospatial attention is not affected during cocaine selfadministration and extinction learning (c), whereas both omissions (d) and response latencies (e) increase during cocaine self-administration and decrease during operant extinction training. Feeder latencies (f) are not affected during cocaine selfadministration and extinction training. $\mathrm{P} 1$ and $\mathrm{P} 2$ indicate baseline performance levels prior to intravenous catheter surgery (P1) and before cocaine selfadministration (P2), respectively. $\mathrm{SA}+\mathrm{PR}$ indicate selfadministration (SA, during all sessions in weeks 1-3) and progressive ratio responding (PR, based on four PR sessions during week 4). $* p<0.05$
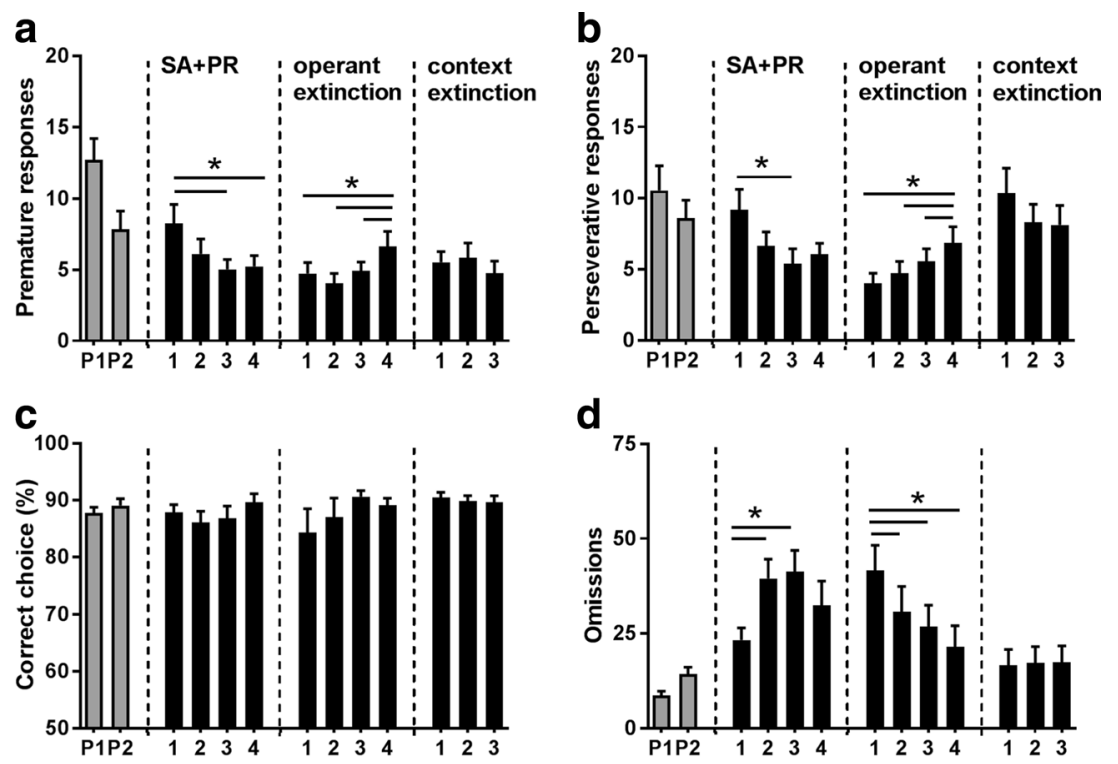

d

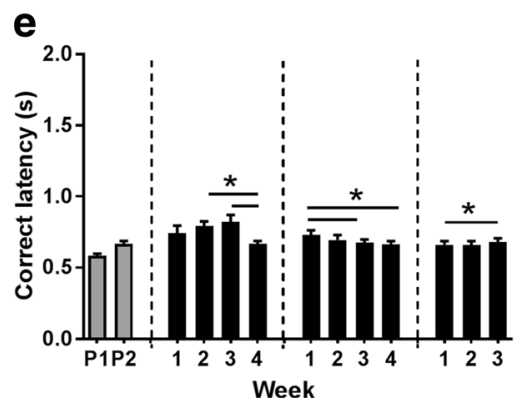

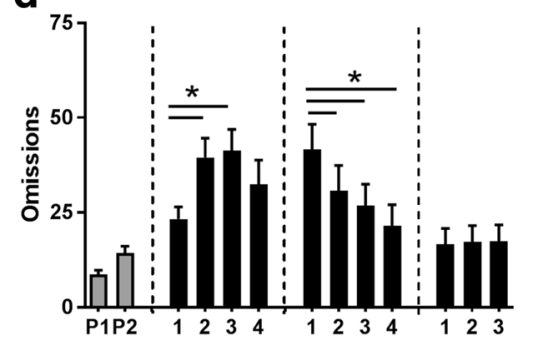

f

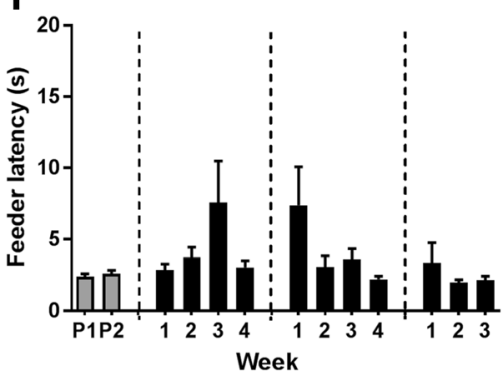




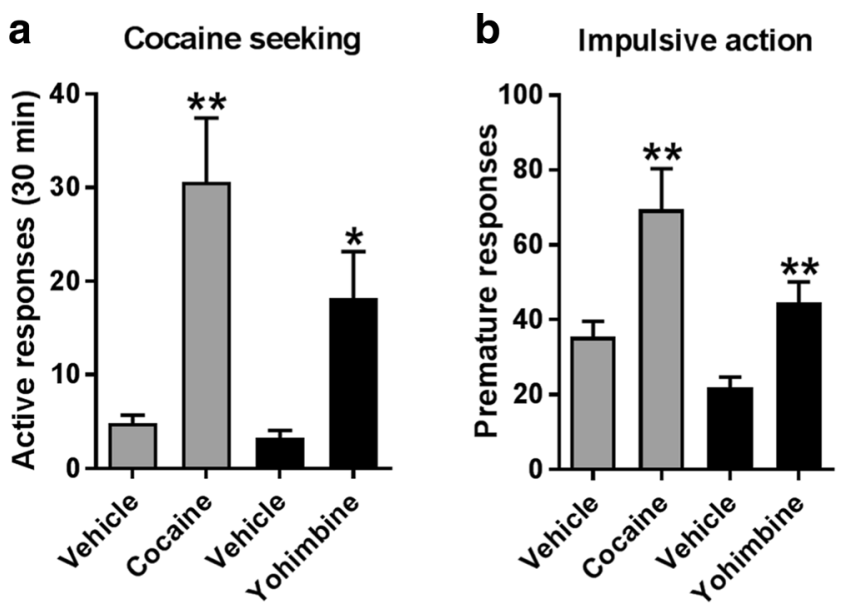

Fig. 4 Acute effects of cocaine and yohimbine on reinstatement to cocaine seeking and on 5-CSRTT performance. Both cocaine $(7.5 \mathrm{mg} / \mathrm{kg})$ and yohimbine $(1.25 \mathrm{mg} / \mathrm{kg})$ induced reinstatement of cocaine seeking (a) and increased impulsive action in the 5-CSRTT (b).

reinstatement were also increased by cocaine from $1.9( \pm 0.5)$ to $4.3( \pm 1.6)$ responses and by yohimbine from $2.1( \pm 0.7)$ to $8.0( \pm 2.5)$ responses [data not shown; dose: $F(1,22)=12.21$, $p=0.002 ;$ drug: $F(1,22)=1.23$, NS; drug $\times$ dose: $F(1,22)=1.42, \mathrm{NS}]$.

Following reinstatement tests, in the 5-CSRTT acute challenges with $7.5 \mathrm{mg} / \mathrm{kg}$ cocaine and $1.25 \mathrm{mg} / \mathrm{kg}$ yohimbine, both strongly increased the number of premature responses compared to their respective vehicles with higher response rates in the cocaine experiments [Fig. $4 \mathrm{~b}$; dose: $F(1,22)=28.43$, $p<0.001$; drug: $F(1,22)=9.41, p=0.006$; drug $\times$ dose: $F(1,22)=1.42$, NS]. Whereas cocaine increased the number of omissions, this measure was not affected by yohimbine [Fig. 4c; dose: $F(1,22)=3.71$, NS; drug: $F(1,22)=4.08$, NS; drug $\times$ dose: $F(1,22)=6.14, p=0.021]$. The levels of accurate choice were reduced by both cocaine and yohimbine [Fig. 4d; dose: $F(1,22)=6.93, p=0.015$; drug: $F(1,22)=2.92$, NS; drug $\times$ dose: $F(1,22)=0.29$, NS]. Lastly, although the effect size was small, response latencies were reduced from $0.61( \pm 0.03)$ to $0.56( \pm 0.04) \mathrm{s}$ by cocaine and from $0.60( \pm 0.03)$ to 0.59 $( \pm 0.03)$ s by yohimbine [data not shown; dose: $F(1,22)=4.49, p=0.046$; drug: $F(1,22)=0.31$, NS; drug $\times$ dose: $F(1,22)=1.98$, NS]. Other measures reflecting task performance in the 5-CSRTT, namely perseverative responses and feeder latencies, were not altered by cocaine or yohimbine [data not shown; all $F<1.13$, NS].

\section{Correlation analyses of the reactivity to cocaine and yohimbine in the 5-CSRTT and cocaine reinstatement}

All correlations between the effects of cocaine and yohimbine on the behavioral measures in the 5-CSRTT and reinstatement of cocaine seeking are depicted in Table 1 . Whereas cocaine increased both the number of premature responses in the 5-

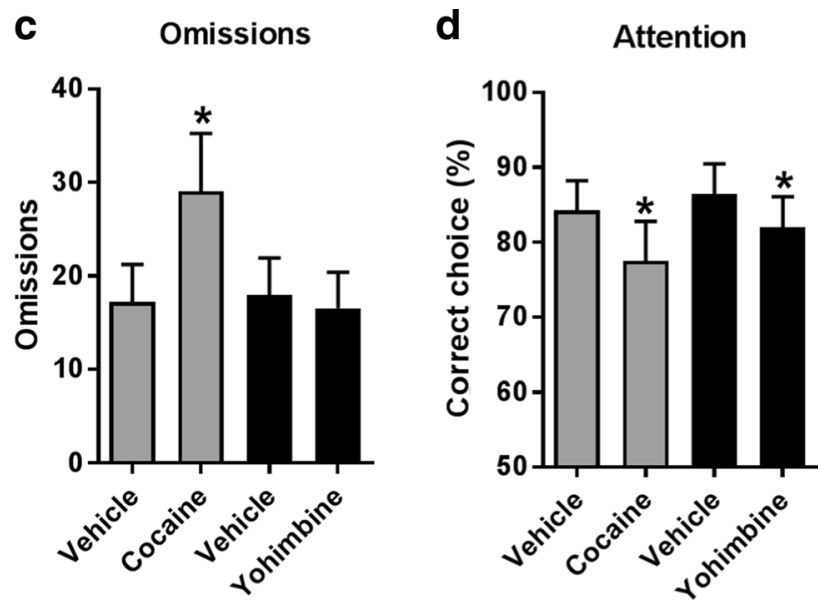

In contrast, only cocaine increased the number of omissions in the 5CSRTT (c), whereas only yohimbine reduced visuospatial attention in the 5-CSRTT (d). $* p<0.05$ and $* * p<0.005$ compared to respective vehicle

CSRTT and active responses during the reinstatement test, these effects were not correlated within individuals [Table 1; Fig. 5a; $R=-0.046$, NS]. However, the reductions in accurate choice induced by cocaine correlated with its effects on active cocaine-seeking responses in the reinstatement test $[R=-0.46$, $p=0.028]$. Also, the increase in the number of omissions in the 5-CSRTT correlated with active cocaine-seeking responses during reinstatement $[R=0.62, p=0.002]$. Moreover, the effects of cocaine on speeding reaction times correlated with active responding in the reinstatement test $[R=-0.48$, $p=0.021]$.

Similar to cocaine, yohimbine-induced premature responding in the 5-CSRTT did not correlate with its effects on the reinstatement of cocaine seeking [Table 1; Fig. 5b; $R=0.058, \mathrm{NS}]$ and neither did any of the other 5-CSRTT parameters.

\section{Discussion}

The present data show that daily intake of cocaine transiently alters impulsive action and measures of attention in the 5CSRTT. Furthermore, acute challenges with both cocaine and the pharmacological stressor yohimbine were found to enhance impulsive action as well as relapse to cocaine seeking, yet these effects showed no individual correlations.

During the course of the entire cocaine SA protocol, rats were concurrently trained in the 5-CSRTT, thereby allowing the assessment of shifts in baseline impulsive action and attentional functioning. Importantly, 5-CSRTT training took place in the mornings about $1 \mathrm{~h}$ prior to cocaine SA training and thus impulsive action was measured under drug-free conditions. 
Table 1 Correlation between cocaine- and yohimbine-induced behavioral effects compared to their respective vehicle on 5CSRTT performance and reinstatement of cocaine seeking

\begin{tabular}{lllll}
\hline & $\begin{array}{l}\Delta \text { Cocaine } \\
\text { active }\end{array}$ & $\begin{array}{l}\Delta \text { Cocaine } \\
\text { inactive }\end{array}$ & $\begin{array}{l}\Delta \text { Yohimbine } \\
\text { active }\end{array}$ & $\begin{array}{l}\Delta \text { Yohimbine } \\
\text { inactive }\end{array}$ \\
\hline$\Delta$ Premature responses & -0.046 & -0.13 & 0.058 & 0.22 \\
$\Delta$ Perseverative responses & -0.22 & 0.38 & -0.048 & -0.12 \\
$\Delta$ Accurate choice & $-0.46^{*}$ & -0.052 & -0.070 & -0.10 \\
$\Delta$ Omissions & $0.62^{*}$ & 0.034 & 0.13 & 0.34 \\
$\Delta$ Correct latency & $-0.48^{*}$ & 0.040 & -0.11 & 0.098 \\
$\Delta$ Feeder latency & -0.21 & 0.10 & 0.20 & 0.35 \\
\hline
\end{tabular}

Indicated are $R$ values

$* p<0.05$
In the present study, long $5 \mathrm{~h}$ access to cocaine induced a transient change in impulsive action with a decrement in the number of premature responses over the weeks of cocaine self-administration. Vice versa, during extinction training, premature responding increased, yet only during the last week of extinction training. As such, the current findings extend previous observations. In this respect, Winstanley and coworkers showed that rats developed a rapid tolerance to the cocaine-induced enhancing effects on premature responding in the 5-CSRTT, whereas withdrawal from cocaine SA resulted in a rapid increase in premature responding (Winstanley et al. 2009). In contrast, no alterations of premature responding were reported earlier during periods of withdrawal in between periods of chronic cocaine self-administration (Dalley et al. 2005). An explanation for these discrepancies might lie in the amount of daily cocaine intake. In the study by Dalley and co-workers, cocaine intake was the highest (8-h sessions, $0.25 \mathrm{mg}$ /infusion), compared to 2 -h sessions and $0.5 \mathrm{mg} / \mathrm{kg} /$ infusion in the study of Winstanley et al. (2009). Our study, with 5-h sessions and $0.5 \mathrm{mg} / \mathrm{kg} /$ infusion would lead to a daily cocaine intake somewhere in between. This would explain the rapid (Winstanley), delayed (present study), and absence of increase in premature responding in the task
(Dalley; not detectable during the 7-day withdrawal test phase).

In the current study, some measures in the 5-CSRTT, reflecting attentional performance and motivation (omissions and latencies, but not accurate choice) showed a clear deterioration over the weeks of cocaine SA which recovered to baseline performance during the extinction period. The previous observations that $2 \mathrm{~h}$ cocaine access only mildly affected omission rate (Winstanley et al. 2009), whereas abstinence from longer ( 5 and $8 \mathrm{~h}$ ) access to cocaine more severely increased omission rates (Dalley et al. 2005), may again indicate that cocaine exposure transiently deteriorates behavioral performance depending on the intake levels of cocaine, which is restored following abstinence.

We found that within the same individuals, acute injections of both cocaine and yohimbine induced an increase in impulsive action as well as an increase in cocaine-seeking responses in the reinstatement tests. However, these drug effects on both behaviors did not correlate with each other within individuals. Thus, although cocaine and yohimbine both induced an increase in impulsive action and drug-seeking behavior, the lack of correlation suggests that the pharmacological effects of stress and a cocaine prime on relapse to cocaine seeking
Fig. 5 Correlation between reactivity to cocaine and yohimbine compared to their respective vehicle on impulsive action in the 5-CSRTT and reinstatement to cocaine seeking. There was no correlation between the effects of both cocaine (a) and yohimbine (b) on the number of premature responses in the 5CSRTT and the number of active cocaine-seeking responses
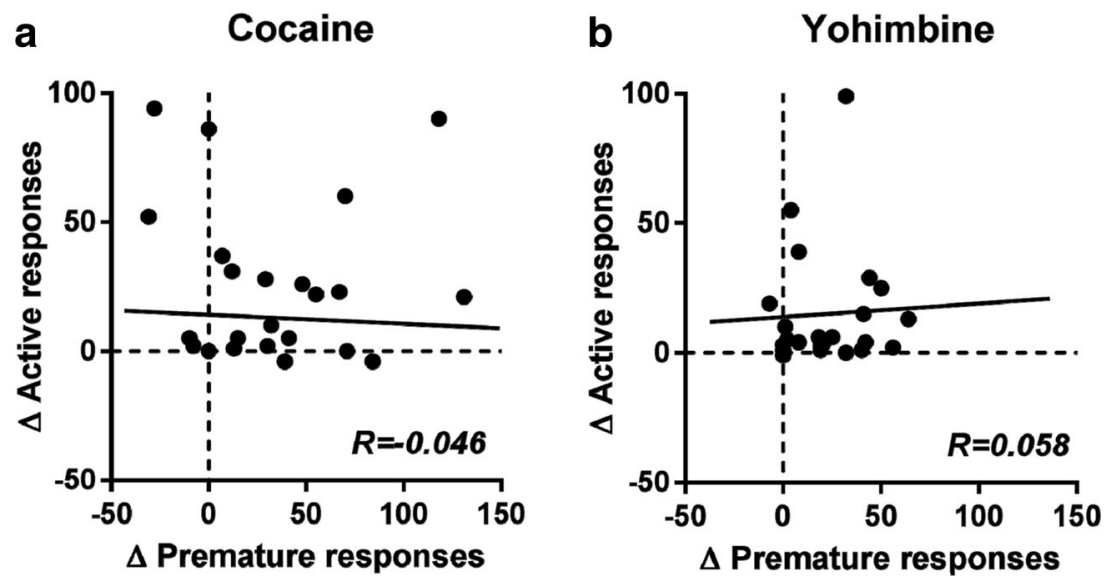
appear not mediated via alterations in "state" impulsive action. Although we did not study the underlying neural mechanisms of cocaine and yohimbine, the current data indicate a different mechanism of action of both cocaine and yohimbine in impulsive action and relapse to cocaine seeking. This notion is supported by previous work. For example, it has been shown that the effects of yohimbine on impulsive action in the 5CSRTT are primarily mediated via the orbitofrontal cortex and not the medial prefrontal cortex nor the nucleus accumbens (Sun et al. 2010). In contrast, the medial prefrontal cortex and nucleus accumbens do play an important role in stressinduced reinstatement to drug-seeking behavior (for a review, see Shaham et al. 2003). In addition, both cocaine-induced increments in impulsive action as well as cocaine-primed reinstatement of drug seeking can be attenuated by a serotonin $2 \mathrm{~A}$ antagonist suggesting a common mechanism for this receptor subtype, yet blocking serotonin $2 \mathrm{C}$ receptors differentially affected these behavioral processes (Fletcher et al. 2002, 2011). Together, such observations question the viability of ameliorating relapse vulnerability via a reduction of impulsive action, and these data extend our previous work on the relationship between impulsive choice, a different modality of impulsivity, and cocaine seeking (Broos et al. 2012a). In that study, both impulsive choice (delayed reward task) and relapse propensity (context-induced relapse) were manipulated with the clinically relevant drug methylphenidate and the dopamine D1 receptor antagonist SCH-23390. Similar to the current observations, we also found no correlation between the changes in impulsive choice and relapse propensity (Broos et al. 2012a). Thus, whereas acute pharmacological challenges modulate both impulsive action and impulsive choice, as well as relapse propensity, the current preclinical evidence suggest that these effects are not correlated within individuals.

These preclinical data are in line with the observed high comorbidity of ADHD and drug dependence in humans, yet the role of ADHD (and its symptomatology) in the etiology of drug dependence is unclear (Lynskey and Hall 2001). Moreover, medication improving ADHD symptoms such as, for instance, methylphenidate does not concurrently attenuate drug craving or relapse to cocaine consumption (Schubiner et al. 2002; for a review, see Pattij and De Vries 2013). This might imply that rehabilitation programs aimed at acute, shortterm reduction of impulsivity will not suffice to maintain treatment retention.

In conclusion, the current observations demonstrate that a history of cocaine consumption alters impulsive action and measures of attention. Importantly, drug-induced increments in state impulsive action appear not to be associated with increments in relapse propensity within the same individuals. Collectively, this may imply that the treatment potential of promoting abstinence via reducing state impulsivity is not straightforward and warrants further investigation.
Acknowledgements We thank Rob Binnekade, Mathijs Stegeman ${ }^{\dagger}$, Yvar van Mourik, and Dustin Schetters for the excellent technical assistance. This work was financially supported by a grant from the Netherlands Organization for Scientific Research (ZonMW grant no. 31160003).

\section{Compliance with ethical standards}

Ethical approval Experiments were approved by the Animal Care committee of the VU University and VU University medical center, Amsterdam, The Netherlands.

Conflict of interest The authors declare that they have no conflict of interest.

Open Access This article is distributed under the terms of the Creative Commons Attribution 4.0 International License (http:// creativecommons.org/licenses/by/4.0/), which permits unrestricted use, distribution, and reproduction in any medium, provided you give appropriate credit to the original author(s) and the source, provide a link to the Creative Commons license, and indicate if changes were made.

\section{References}

Belin D, Mar AC, Dalley JW, Robbins TW, Everitt BJ (2008) High impulsivity predicts the switch to compulsive cocaine-taking. Science 320:1352-1355

Bossert JM, Ghitza UE, Lu L, Epstein DH, Shaham Y (2005) Neurobiology of relapse to heroin and cocaine seeking: an update and clinical implications. Eur J Pharmacol 526:36-50

Broos N, Diergaarde L, Schoffelmeer AN, Pattij T, De Vries TJ (2012a) Trait impulsive choice predicts resistance to extinction and propensity to relapse to cocaine seeking: a bidirectional investigation. Neuropsychopharmacology 37:1377-1386

Broos N, Schmaal L, Wiskerke J, Kostelijk L, Lam T, Stoop N, Weierink L, Ham J, de Geus EJ, Schoffelmeer AN, van den Brink W, Veltman DJ, De Vries TJ, Pattij T, Goudriaan AE (2012b) The relationship between impulsive choice and impulsive action: a cross-species translational study. PLoS One 7:e36781

Broos N, Loonstra R, van Mourik Y, Schetters D, Schoffelmeer AN, Pattij T, De Vries TJ (2015) Subchronic administration of atomoxetine causes an enduring reduction in contextinduced relapse to cocaine seeking without affecting impulsive decision making. Addict Biol 20:714-723

Caprioli D, Hong YT, Sawiak SJ, Ferrari V, Williamson DJ, Jupp B, Adrian CT, Aigbirhio FI, Everitt BJ, Robbins TW, Fryer TD, Dalley JW (2013) Baseline-dependent effects of cocaine preexposure on impulsivity and $\mathrm{D}(2 / 3)$ receptor availability in the rat striatum: possible relevance to the attention-deficit hyperactivity syndrome. Neuropsychopharmacology 38:1460-1471

Charney DS, Heninger GR, Breier A (1984) Noradrenergic function in panic anxiety. Effects of yohimbine in healthy subjects and patients with agoraphobia and panic disorder. Arch Gen Psychiatry 41:751763

Dalley JW, Laane K, Pena Y, Theobald DE, Everitt BJ, Robbins TW (2005) Attentional and motivational deficits in rats withdrawn from intravenous self-administration of cocaine or heroin. Psychopharmacology 182:579-587

Dalley JW, Fryer TD, Brichard L, Robinson ES, Theobald DE, Laane K, Pena Y, Murphy ER, Shah Y, Probst K, Abakumova I, Aigbirhio FI, Richards HK, Hong Y, Baron JC, Everitt BJ, Robbins TW (2007) 
Nucleus accumbens D2/3 receptors predict trait impulsivity and cocaine reinforcement. Science 315:1267-1270

De Wit H (2009) Impulsivity as a determinant and consequence of drug use: a review of underlying processes. Addict Biol 14:22-31

Diergaarde L, Pattij T, Poortvliet I, Hogenboom F, de Vries W, Schoffelmeer AN, De Vries TJ (2008) Impulsive choice and impulsive action predict vulnerability to distinct stages of nicotine seeking in rats. Biol Psychiatry 63: 301-308

Economidou D, Pelloux Y, Robbins TW, Dalley JW, Everitt BJ (2009) High impulsivity predicts relapse to cocaine-seeking after punishment-induced abstinence. Biol Psychiatry 65:851-856

Evenden JL (1999) Varieties of impulsivity. Psychopharmacology 146: 348-361

Fletcher PJ, Grottick AJ, Higgins GA (2002) Differential effects of the 5 $\mathrm{HT}(2 \mathrm{~A})$ receptor antagonist M100907 and the 5-HT(2C) receptor antagonist SB242084 on cocaine-induced locomotor activity, cocaine self-administration and cocaine-induced reinstatement of responding. Neuropsychopharmacology 27:576-586

Fletcher PJ, Rizos Z, Noble K, Higgins GA (2011) Impulsive action induced by amphetamine, cocaine and MK801 is reduced by 5$\mathrm{HT}(2 \mathrm{C})$ receptor stimulation and 5-HT(2A) receptor blockade. Neuropharmacology 61:468-477

van Gaalen MM, Brueggeman RJ, Bronius PF, Schoffelmeer AN, Vanderschuren LJ (2006) Behavioral disinhibition requires dopamine receptor activation. Psychopharmacology 187:73-85

Van Emmerik-van Oortmerssen K, Van de Glind G, Van den Brink W, Smit F, Crunelle CL, Swets M, Schoevers RA (2012) Prevalence of attention-deficit hyperactivity disorder in substance use disorder patients: a meta-analysis and meta-regression analysis. Drug Alcohol Depend 122:11-19

Jupp B, Caprioli D, Dalley JW (2013). Highly impulsive rats: modelling an endophenotype to determine the neurobiological, genetic and environmental mechanisms of addiction. Dis Model Mech 6:302311

Lynskey MT, Hall W (2001) Attention deficit hyperactivity disorder and substance use disorders: is there a causal link? Addiction 96:815822

Mantsch JR, Baker DA, Funk D, Lê AD, Shaham Y (2016) Stressinduced reinstatement of drug seeking: 20 years of progress. Neuropsychopharmacology 41:335-356

Moeller FG, Barratt ES, Dougherty DM, Schmitz JM, Swann AC (2001) Psychiatric aspects of impulsivity. Am J Psychiatry 158:1783-1793

Pattij T, De Vries TJ (2013) The role of impulsivity in relapse vulnerability. Curr Opin Neurobiol 23:700-705
Perry JL, Carroll ME (2008) The role of impulsive behavior in drug abuse. Psychopharmacology 200:1-26

Schippers MC, Schetters D, De Vries TJ, Pattij T (2016) Differential effects of the pharmacological stressor yohimbine on impulsive decision making and response inhibition. Psychopharmacology 233: 2775-2785

Schubiner H, Saules KK, Arfken CL, Johanson CE, Schuster CR, Lockhart N, Edwards A, Donlin J, Pihlgren E (2002) Double-blind placebo-controlled trial of methylphenidate in the treatment of adult ADHD patients with comorbid cocaine dependence. Exp Clin Psychopharmacol 10:286-294

Shaham Y, Shalev U, Lu L, De Wit H, Stewart J (2003) The reinstatement model of drug relapse: history, methodology and major findings. Psychopharmacology 168:3-20

Shepard JD, Bossert JM, Liu SY, Shaham Y (2004) The anxiogenic drug yohimbine reinstates methamphetamine seeking in a rat model of drug relapse. Biol Psychiatry 55:1082-1089

Sun H, Green TA, Theobald DE, Birnbaum SG, Graham DL, Zeeb FD, Nestler EJ, Winstanley CA (2010) Yohimbine increases impulsivity through activation of cAMP response element binding in the orbitofrontal cortex. Biol Psychiatry 67:649-656

Torregrossa MM, Xie M, Taylor JR (2012) Chronic corticosterone exposure during adolescence reduces impulsive action but increases impulsive choice and sensitivity to yohimbine in male SpragueDawley rats. Neuropsychopharmacology 37:1656-1670

Verdejo-Garcia A, Lawrence AJ, Clark L (2008) Impulsivity as a vulnerability marker for substance-use disorders: review of findings from high-risk research, problem gamblers and genetic association studies. Neurosci Biobehav Rev 32:777-810

Winstanley CA, Dalley JW, Theobald DEH, Robbins TW (2004) Fractionating impulsivity: contrasting effects of central 5-HT depletion on different measures of impulsive behavior. Neuropsychopharmacology 29:1331-1343

Winstanley CA, LaPlant Q, Theobald DE, Green TA, Bachtell RK, Perrotti LI, DiLeone RJ, Russo SJ, Garth WJ, Self DW, Nestler EJ (2007) DeltaFosB induction in orbitofrontal cortex mediates tolerance to cocaine-induced cognitive dysfunction. J Neurosci 27: 10497-10507

Winstanley CA, Bachtell RK, Theobald DE, Laali S, Green TA, Kumar A, Chakravarty S, Self DW, Nestler EJ (2009) Increased impulsivity during withdrawal from cocaine self-administration: role for $\{$ Delta $\}$ FosB in the orbitofrontal cortex. Cereb Cortex 19:435-444

Winstanley CA, Olausson P, Taylor JR, Jentsch JD (2010) Insight into the relationship between impulsivity and substance abuse from studies using animal models. Alcohol Clin Exp Res 34:1306-1318 